\title{
Article
}

\section{Dynamical friction and the distribution of dark matter in barred galaxies}

Debattista, Victor P and Sellwood, J A

Available at https://clok.uclan.ac.uk/17022/

Debattista, Victor P orcid iconORCID: 0000-0001-7902-0116 and Sellwood, J A (1998) Dynamical friction and the distribution of dark matter in barred galaxies. The Astrophysical Journal, 493 . L5-L8. ISSN 0004-637X

It is advisable to refer to the publisher's version if you intend to cite from the work.

For more information about UCLan's research in this area go to

http://www.uclan.ac.uk/researchgroups/ and search for < name of research Group>.

For information about Research generally at UCLan please go to

http://www.uclan.ac.uk/research/

All outputs in CLoK are protected by Intellectual Property Rights law, including Copyright law. Copyright, IPR and Moral Rights for the works on this site are retained by the individual authors and/or other copyright owners. Terms and conditions for use of this material are defined in the policies page.

\section{CLoK}

Central Lancashire online Knowledge www.clok.uclan.ac.uk

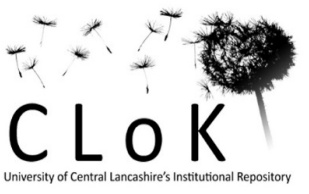




\title{
DYNAMICAL FRICTION AND THE DISTRIBUTION OF DARK MATTER IN BARRED GALAXIES
}

\author{
Victor P. Debattista and J. A. Sellwood \\ Department of Physics and Astronomy, Rutgers University, Box 0849 Piscataway, NJ 08855-0849; debattis@physics.rutgers.edu \\ Received 1997 May 23; accepted 1997 November 12; published 1998 January 6
}

\begin{abstract}
We use fully self-consistent $N$-body simulations of barred galaxies to show that dynamical friction from a dense dark matter halo dramatically slows the rotation rate of bars. Our result supports previous theoretical predictions for a bar rotating within a massive halo. On the other hand, low-density halos, such as those required for maximum disks, allow the bar to continue to rotate at a high rate. There is somewhat meager observational evidence indicating that bars in real galaxies do rotate rapidly, and we use our result to argue that dark matter halos must have a low central density in all high surface brightness disk galaxies, including the Milky Way. Bars in galaxies that have larger fractions of dark matter should rotate slowly, and we suggest that a promising place to look for such candidate objects is among galaxies of intermediate surface brightness.
\end{abstract}

Subject headings: galaxies: evolution — galaxies: halos — galaxies: kinematics and dynamics — Galaxy: halo - Galaxy: structure

\section{INTRODUCTION}

The failure of disk galaxy rotation curves to decline significantly at large radii (see, e.g., Rubin, Ford, \& Thonnard 1980; Bosma 1981) is usually interpreted as evidence that the luminous disk is embedded within a more extensive halo of dark matter (DM). Decomposition of rotation curves into separate contributions from luminous and dark matter is an issue of some controversy, however.

One generally assigns a constant mass-to-light ratio, $M / L$, to the disk (and bulge, if treated separately) and varies parameters of the assumed DM distribution to obtain the best fit to the observed rotation curve. The controversy has arisen because this procedure is very poorly constrained. The $M / L$-values of the luminous matter are uncertain and the fitted functional form for the halo is generally chosen arbitrarily (see, e.g., Broeils 1992, but see also Navarro, Frenk, \& White 1996). Moreover, few galaxies manifest a feature in the shape of the rotation curve to mark the radius at which the dominant contribution to the central attraction switches from luminous to dark matter. Some argue that the DM contribution is substantial at all radii (see, e.g., van der Kruit 1995), while others (see, e.g., van Albada \& Sancisi 1986; Freeman 1992) argue for the so-called maximum disk hypothesis, which requires the largest possible mass in luminous matter consistent with not exceeding the inner rotation curve. Maximum disks are motivated by the fact that luminous matter alone generally accounts well for overall shapes of rotation curves in the inner parts of bright galaxies (Kalnajs 1983; Kent 1986; Buchhorn 1992; Palunas 1996).

In this Letter, we introduce a new argument, quite separate from rotation curve fitting, to bear on the question of the DM content of disk galaxies: a rapidly rotating bar within a massive halo must lose angular momentum to the halo through dynamical friction. Sellwood (1980) reported a secular gain of angular momentum by the halo in a low-quality simulation of a barred galaxy, and this effect had been predicted by Tremaine (1975, unpublished). Weinberg (1985) estimated the bar deceleration rate using linear perturbative calculations and simple models for the bar and halo, finding that the bar would slow down on the surprisingly short timescale of a few rotation periods. Until recently, this prediction had not been thoroughly tested: simulations by Combes et al. (1990) had only a bulge of live particles, not an extensive halo; Raha et al. (1991) did not evolve their models for long enough; the "halo" particles employed by Little \& Carlberg (1991) were confined to a plane, and the bar used by Hernquist \& Weinberg (1992) was rigid and their model had no disk. Our realistic simulations (Debattista \& Sellwood 1996), and those of Athanassoula (1996), have confirmed that self-consistent bars are rapidly braked by a massive responsive halo.

In order to relate theoretical work to real galaxies, we use the dimensionless ratio $D_{L} / a_{B}$ to characterize the rate of rotation of a bar. Here $D_{L}$ is the distance from the center of the galaxy to the Lagrange point on the bar major axis (corotation) and $a_{B}$ is the semimajor axis of the bar. This ratio is expected to exceed unity in self-consistent stellar bars (Contopoulos 1980) and is found to be not much larger for the bars that form in most $N$-body simulations of bar unstable disks (see, e.g., Sellwood 1981; Combes \& Sanders 1981). We describe as slow bars those for which $D_{L} / a_{B}$ is substantially greater than unity.

The determination of this parameter for galaxies ideally requires a direct measurement of the bar pattern speed, $\Omega_{p}$. Merrifield \& Kuijken (1995) estimate $D_{L} / a_{B}=1.4 \pm 0.3$ for the SB0 galaxy NGC 936. Indirect estimates can be obtained by matching hydrodynamical simulations with observations; Athanassoula's (1992) comparison of shocks in two-dimensional gas flows with the locations and shapes of dust lanes in barred galaxies led her to suggest that $D_{L} / a_{B}=1.2 \pm 0.2$. Studies of specific galaxies (Lindblad, Lindblad, \& Athanassoula 1996; Weiner et al. 1996), using potentials derived from the observed near infrared light distribution, yield similar values. While the evidence is meager, it does suggest that $D_{L} / a_{B} \lesssim 1.5$ for Hubble types SB0 through SBc.

Our simulations show that, as predicted, bars rotating inside a halo of moderate central density slow down in a short time, yielding $D_{L} / a_{B}>1.5$. It seems possible to avoid an inconsistency with the observed properties of galaxies only by reducing the halo central density to the point where the luminous matter dominates the rotation curve throughout most of the disk.

\section{NUMERICAL TECHNIQUE}

We create fully self-consistent $N$-body models of disk-halo galaxies to simulate their evolution. We have employed two different types of grid, having both Cartesian and cylindrical polar geometries, and upward of 300,000 particles. We have 

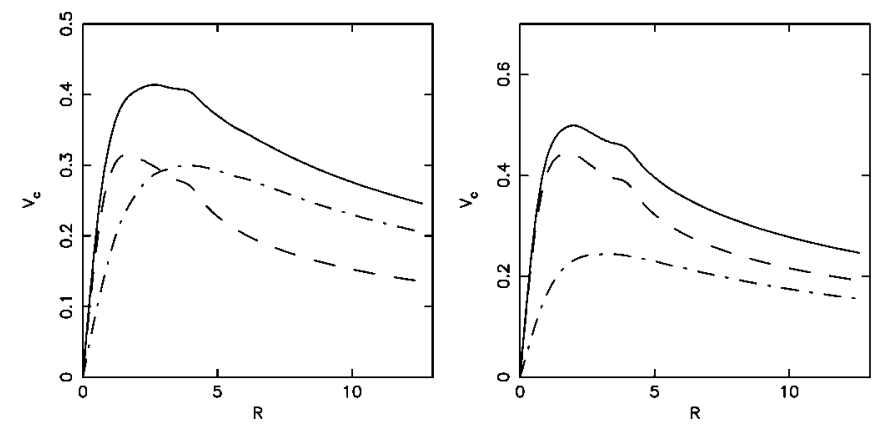

FIG. 1.-Rotation curves for the most massive halo (left) and the least massive halo (right) from the series of models without extensive halos. The curves are decomposed into disk (dashed line) and halo (dot-dashed line) contributions. The total mass is the same in both models; the disk mass fractions are, respectively, 0.3 and 0.6 in the left and right panels.

verified that results are insensitive to the numerical scheme and are unchanged when $N$ is increased by a factor of 5 .

Our initially axisymmetric equilibrium models were created by an extension of the procedure first employed by Raha et al. (1991) that takes into account the combined gravitational fields of the disk and halo. The halo particles in all the models we present in this Letter are selected from a nonrotating, isotropic distribution function (DF) of polytropic form. The initial disks have velocity dispersions set by adopting a constant Toomre $Q$ parameter and thickness at all radii. A fuller description of our numerical procedures will be given in a later paper.

\section{SIMULATION RESULTS}

To illustrate our main point, we first present a series of models with halos that do not extend to large radii. These experiments were run on modest grids, which cannot contain extensive halos if they are to afford reasonable resolution for the disk. We later present a model run on a much larger grid to demonstrate that a more realistic halo does not alter our conclusion.

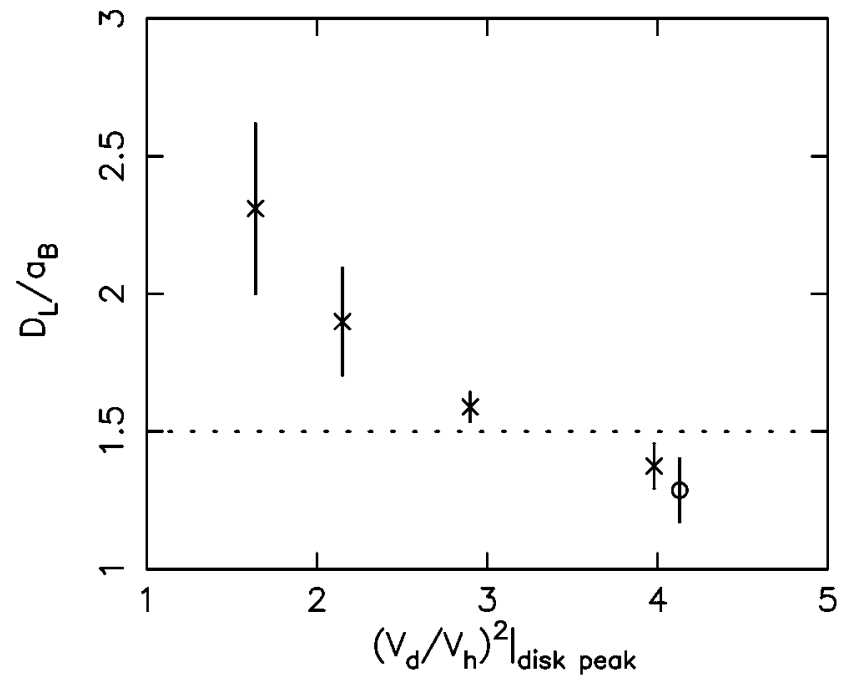

FIG. 2.-Final equilibrium $D_{L} / a_{B}$ plotted against the square of the initial ratio of disk to halo circular velocities, evaluated at the peak of the disk rotation curve. The error bars are calculated from time averages. The dotted horizontal line is at $D_{L} / a_{B}=1.5$. The different symbols are explained in the text.

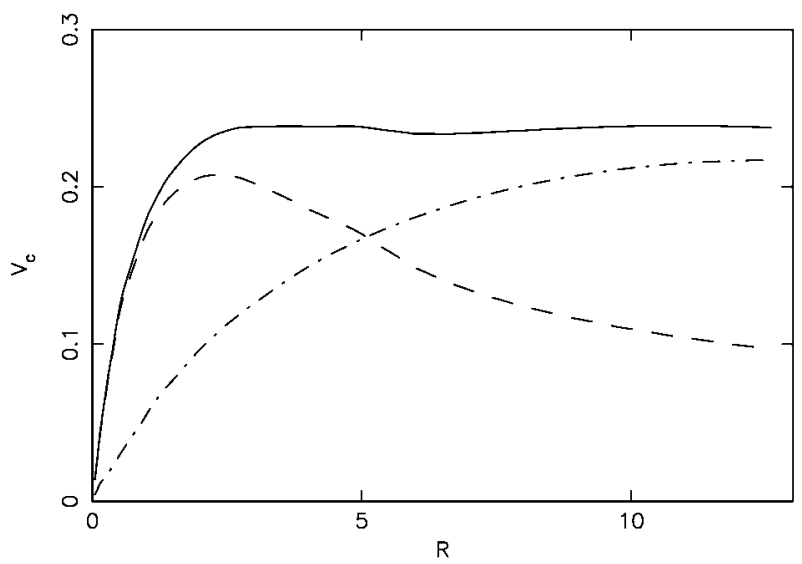

FIG. 3.-Rotation curve for the maximum disk model decomposed into disk (dashed line) and halo (dot-dashed line) contributions.

\subsection{Models without Extensive Halos}

The rotation curves for two models, together with the separate contributions of the disk and halo, are shown in Figure 1. These models have the greatest and least massive "halos" of a series of four models run on moderate size grids; the only property that varies along this sequence is the disk/halo mass ratio. In none of the models is the halo dense enough to suppress the bar instability in the disk. All quickly form strong, rapidly rotating bars that subsequently bend and thicken in the usual way (Combes et al. 1990; Raha et al. 1991).

As predicted, as soon as the disk is strongly nonaxisymmetric, the bars begin to lose angular momentum through dynamical friction. The rotating bar quickly induces a bisymmetric distortion in the distribution of halo particles that lags the bar by up to $\sim 45^{\circ}$; the consequent torque gradually decreases as the distortion in the halo becomes aligned with the bar.

In the model with the most massive halo, angular momentum transfer ceases after about 50 initial rotation periods of the bar, by which time the halo has gained some $40 \%$ of the disk's initial angular momentum. As the bar loses angular momentum, its pattern speed drops-by a factor of 5 in this model-in agreement with Weinberg's (1985) conclusion that bars can be rapidly braked by dynamical friction.

The bar in the model with the least massive halo, on the other hand, continues to rotate quite rapidly. Bar deceleration is less for two reasons: (1) a rather mild distortion in the lower density halo has a major axis that lags the bar by only $\sim 10^{\circ}$ creating a much weaker torque, and (2) the disk, and therefore the bar, mass is greater so that the pattern speed changes produced by the weaker torque are much less.

The ratio $D_{L} / a_{B}$ increases steadily as the bars slow down. The crosses in Figure 2 show our main result from this series of models, that the final value of $D_{L} / a_{B}$ increases systematically with the mass of the halo. Only our model with the extreme low-mass halo has $D_{L} / a_{B} \lesssim 1.5$ and remains in the range consistent with the observed values discussed in the Introduction.

\subsection{A More Realistic Model}

Since these models have unrealistic halos, we have run a further experiment on a much larger grid in which the rotation curve is flat over a wide range of radii, as shown in Figure 3. Once again the halo was a simple, nonrotating polytrope with 
an isotropic distribution of velocities, but here we adjusted the halo mass and outer cutoff radius to give us a maximum disk model.

The behavior of this much more realistic case is very similar to that of the low-mass halo model from the earlier sequence. The disk quickly formed a strong bar that slowed only slightly; the bar pattern speed stabilized some $25 \%$ below its initial value as angular momentum loss to the halo dropped to a very low rate. The ratio $D_{L} / a_{B}$, shown by the circle in Figure 2 , settled to about 1.3 after just three bar periods and remained at this value for the next 12 bar rotations with no hint of an increase.

The central halo density in this model is low; at the peak of the disk rotation curve $V_{\text {disk }} / V_{\text {halo }}$ is about the same as in the model with the lowest density halo in the sequence described in $\S 3.1$. Since the resulting value for $D_{L} / a_{B}$ is also quite similar, we argue that dynamical friction is determined by the density of the inner halo and not the total halo mass. This conclusion is in agreement with previous wisdom (Binney \& Tremaine $1987, \S 7.1)$ that dynamical friction in an inhomogeneous stellar system depends upon the local mass density at the position of the perturber.

It should be noted that our chosen parameter, $\left(V_{\text {disk }} / V_{\text {halo }}\right)^{2}$ at some radius is a measure of the interior halo mass and not the local density. The critical value may therefore depend upon the density profile of the inner halo.

\subsection{Other Models}

We have run many more models to experiment with rotating and anisotropic halos and have found that friction is not lessened dramatically for any reasonable halo DF. Making more halo particles orbit in the same sense as the disk does reduce friction, but even giving almost all halo particles the same sign of angular momentum still led eventually to an unacceptably slow bar in our densest halo. In no case were the effects of secondary bar growth (Sellwood 1981) enough to compensate for the bar deceleration. We give further details of these tests elsewhere.

\section{DISCUSSION}

The stellar dynamical models just described exclude possible mechanisms that could speed up bars. Gas is driven inward by angular momentum loss to the bar, but it seems unrealistic to suggest that enough could be gained from gas to compensate for the $40 \%$ lost by the disk in our densest halo case. More importantly, mass inflows lead to a secular increase in the central density that will cause the pattern speed to rise (Kalnajs 1996). Taken to excess, however, too great a mass buildup in the center will destroy a bar (Norman, Sellwood, \& Hassan 1996). Note also that gas driven changes of this kind cannot occur in SB0 galaxies such as NGC 936, which is known to have a fast bar.

If bars were short-lived, they could dissolve before they have time to slow down. We do not regard this idea as attractive for a number of reasons. First, to avoid any slow cases, bars would have to be destroyed relatively soon after they formed. Second, unless all bars have recently formed for the first time (implausible), the large fraction of disk galaxies that are strongly barred ( $30 \%$, Sellwood \& Wilkinson 1993) would require short-lived bars to recur in every galaxy. Bar dissolution (through a growing central mass or a minor merger, say) would leave the stellar disk hot and unresponsive, and it seems inconceivable that disks could repeatedly recover to allow rapid redevelopment of fresh bars. Third, the bars in all our simulations are very robust and survive even a fivefold reduction in pattern speed.

Weinberg (1985) suggested that the disk may be able to transfer angular momentum to the bar at an inner Lindblad resonance, keeping it in rapid rotation. Previous simulations (see, e.g., Sellwood 1981), as well as those described here, show that the net exchange of angular momentum between the bar and the disk amounts to a loss by the bar. It does not seem possible that a rapidly rotating bulge could torque up the bar either: even when we gave near maximal rotation to our densest halo simulation, in which some fraction of the halo mass could be thought of as a rapidly rotating bulge, the bar still slowed unacceptably.

\subsection{The Milky Way}

The contribution of DM to the circular velocity at the solar position is still a matter of debate (Kuijken \& Gilmore 1991; Merrifield 1993; Sellwood \& Sanders 1988; Sackett 1997). There is also some disagreement over the precise size (Weinberg 1992; Dwek et al. 1995) and pattern speed of the bar (Binney et al. 1991; Kalnajs 1996), but it seems unlikely that the value of $D_{L} / a_{B}$ will turn out to be substantially larger than for other barred galaxies. Verification that the bar in the Milky Way is indeed fast would strengthen the arguments in favor of a maximum disk.

\subsection{Unbarred Galaxies}

We have argued that barred galaxies must have maximum disks in order that the bar rotation rate remain consistent with the believed properties of barred galaxies. Here we extend the argument to unbarred galaxies. There are no qualitative differences between the outer rotation curve shapes of SB and of SA galaxies (Bosma 1996), which suggests that their DM mass distributions are quite similar. Ostriker \& Peebles (1973) argued that the survival of an unbarred disk requires a galaxy to be DM dominated, implying that the disks in SA galaxies should be less than maximum. If we accept their argument for the moment, and also the seemingly reasonable proposition that there is a continuum of DM content from SA to SB galaxies, then we are forced to conclude that there should be some barred galaxies with enough halo to brake the bar but not enough to suppress it, as in our models. The apparent absence of slow bars in high surface brightness galaxies implies that there is no continuum between high and low-density halos. We find a single, maximum-disk distribution to be more natural than a bimodal distribution of halo fractions, and some other mechanism must be responsible for the stability of unbarred galaxies (see, e.g., Binney \& Tremaine 1987, § 6.3.2).

\subsection{Prospects for Finding Slow Bars}

The newly discovered class of low surface brightness (LSB) galaxies (see Bothun, Impey, \& McGaugh 1997 for a review) exhibits the same Tully-Fisher relation as do high surface brightness (HSB) galaxies (Zwaan et al. 1995) and therefore must contain larger fractions of DM (de Blok \& McGaugh 1996). Any bars in galaxies of low or intermediate surface brightness with significant DM content should have been slowed by dynamical friction. The absence of known slow bars may simply reflect an observational bias in favor of HSB galaxies. 


\section{CONCLUSION}

We have argued that the apparent absence of slow bars in HSB galaxies indicates that DM should not have a high central density. We find that dynamical friction from slowly rotating, isotropic halos is unacceptably strong when the halo makes a major contribution to the circular speed at about two disk scale lengths-i.e., within the region where bars are found. It is clearly desirable to determine pattern speeds of many more bars, and we suggest that promising candidates for slow bars might be found amongst galaxies of low or intermediate surface brightness.

We thank an anonymous referee for constructive comments. This work was supported by NSF grant AST 93/18617 and NASA Theory grant NAG 5-2803.

\section{REFERENCES}

Athanassoula, E. 1992, MNRAS, 259, 345

1996, in IAU Colloq. 157, Barred Galaxies, ed. R. Buta, D. A. Crocker, \& B. G. Elmegreen (San Francisco: ASP), 309

Binney, J., Gerhard, O., Stark, A., Bally, J., \& Uchida, K. 1991, MNRAS, 252,210

Binney, J., \& Tremaine, S. 1987, Galactic Dynamics (Princeton: Princeton Univ. Press)

Bosma, A. 1981, AJ, 86, 1791

1996, in IAU Colloq. 157, Barred Galaxies, ed. R. Buta, D. A. Crocker, \& B. G. Elmegreen (San Francisco: ASP), 132

Bothun, G., Impey, C., \& McGaugh, S. 1997, PASP, 109, 745

Broeils, A. 1992, Ph.D. Thesis, Groningen Univ.

Buchhorn, M. 1992, Ph.D. thesis, Australian National Univ.

Combes, F., Debbasch, F., Friedli, D., \& Pfenniger, D. 1990, A\&A, 233, 82

Combes, F., \& Sanders, R. H. 1981, A\&A, 96, 164

Contopoulos, G. 1980, A\&A, 81, 198

Debattista, V. P., \& Sellwood, J. A. 1996, in IAU Colloq. 157, Barred Galaxies, ed. R. Buta, D. A. Crocker, \& B. G. Elmegreen (San Francisco: ASP), 357 de Blok, W. J. G., \& McGaugh, S. S. 1996, ApJ, 469, L89

Dwek, E., Arendt, R. G., Hauser, M. G., Kelsall, T., Lisse, C. M., Moseley, S. H., Silverberg, R. F., Sodroski, T. J., \& Weiland, J. L. 1995, ApJ, 445, 716

Freeman, K. C. 1992, in Physics of Nearby Galaxies: Nature or Nurture?, ed. T. X. Thuan, C. Balkawski, \& J. T. T. Van (Gif-sur-Yvette: Editions Frontières), 201

Hernquist, L., \& Weinberg, M. D. 1992, ApJ, 400, 80

Kalnajs, A. J. 1983, in Internal Kinematics and Dynamics of Galaxies, ed. E. Athanassoula (Dordrecht: Reidel), 87

1996, in Barred Galaxies and Circumnuclear Activity, ed. Aa. Sandqvist, \& P. O. Lindblad (Berlin: Springer), 165
Kent, S. M. 1986, AJ, 91, 1301

Kuijken, K., \& Gilmore, G. 1991, ApJ, 367, L9

Lindblad, P. A. B., Lindblad, P. O., \& Athanassoula, E. 1996, A\&A, 313, 65

Little, B., \& Carlberg, R. G. 1991, MNRAS, 250, 161

Merrifield, M. R. 1993, in Back to the Galaxy, ed. S. S. Holt, \& F. Verter (New York: AIP) 437

Merrifield, M. R., \& Kuijken, K. 1995, MNRAS, 274, 933

Navarro, J. F., Frenk, C. S., \& White, S. D. M. 1996, ApJ, 462, 563

Norman, C. A., Sellwood, J. A., \& Hasan, H. 1996, ApJ, 462, 114

Ostriker, J. P., \& Peebles, P. J. E. 1973, ApJ, 186, 467

Palunas, P. 1996, Ph.D. thesis, Rutgers Univ.

Raha, N., Sellwood, J. A., James, R. A., \& Kahn, F. D. 1991, Nature, 352, 411

Rubin, V. C., Ford, W. K., \& Thonnard, N. 1980, ApJ, 238, 471

Sackett, P. D. 1997, ApJ, 483, 103

Sellwood, J. A. 1980, A\&A, 89, 296 . 1981, A\&A, 99, 362

Sellwood, J. A., \& Sanders, R. H. 1988, MNRAS, 233, 611

Sellwood, J. A., \& Wilkinson, A. 1993, Rep. Prog. Phys., 56, 173

van Albada, G. D., \& Sancisi, R. 1986, Philos. Trans. R. Soc. London A, 320, 447

van der Kruit, P. C. 1995, in IAU Symp. 164, Stellar Populations, ed. P. C. van der Kruit, \& G. Gilmore (Dordrecht: Kluwer), 205

Weinberg, M. D. 1985, MNRAS, 213, 451 . 1992, ApJ, 392, L67

Weiner, B. J., Sellwood, J. A., \& Williams, T. B. 1996, in IAU Colloq. 157, Barred Galaxies, ed. R. Buta, D. A. Crocker, \& B. G. Elmegreen (San Francisco: ASP), 489

Zwaan, M. A., van der Hulst, J. M., de Blok, W. J. G., \& McGaugh, S. S. 1995, MNRAS, 273, L35 